\section{(6) OPEN ACCESS}

\title{
Preoperative next-generation sequencing of pancreatic cyst fluid is highly accurate in cyst classification and detection of advanced neoplasia
}

\author{
Aatur D Singhi, ${ }^{1}$ Kevin McGrath, ${ }^{2}$ Randall E Brand, ${ }^{2}$ Asif Khalid, ${ }^{2}$ Herbert J Zeh, ${ }^{3}$ \\ Jennifer S Chennat, ${ }^{2}$ Kenneth E Fasanella, ${ }^{2}$ Georgios I Papachristou, ${ }^{2}$ Adam Slivka, ${ }^{2}$ \\ David L Bartlett, ${ }^{3}$ Anil K Dasyam, ${ }^{4}$ Melissa Hogg, ${ }_{1}^{3}$ Kenneth K Lee, ${ }^{3}$ \\ James Wallis Marsh, ${ }^{3}$ Sara E Monaco, ${ }^{1}$ N Paul Ohori, ${ }^{1}$ James F Pingpank, ${ }^{3}$ Allan Tsung, ${ }^{3}$ \\ Amer H Zureikat, ${ }^{3}$ Abigail I Wald, ${ }^{1}$ Marina N Nikiforova ${ }^{1}$
}

- Additional material for this paper are available online. To view please visit the journal (http://dx.doi.org/10.1136/ gutjnl-2016-313586).

${ }^{1}$ Department of Pathology, University of Pittsburgh Medical Center Health System, Pittsburgh, Pennsylvania, USA Department of Medicine, University of Pittsburgh, Pittsburgh, Pennsylvania, USA ${ }^{3}$ Department of Surgery, University of Pittsburgh Medical Center, Pittsburgh, Pennsylvania, USA

${ }^{4}$ Department of Radiology، University of Pittsburgh, Pittsburgh, Pennsylvania, USA

\section{Correspondence to}

Dr Aatur D Singhi, Department of Pathology, University of Pittsburgh Medical Center Health System, Pittsburgh, Pennsylvania, PA 15213, USA; singhiad@upmc.edu

Received 13 December 2016 Revised 29 August 2017

Accepted 14 September 2017 Published Online First 28 September 2017

\section{ABSTRACT}

Objective DNA-based testing of pancreatic cyst fluid (PCF) is a useful adjunct to the evaluation of pancreatic cysts (PCS). Mutations in KRAS/GNAS are highly specific for intraductal papillary mucinous neoplasms (IPMNs) and mucinous cystic neoplasms (MCNs), while TP53/PIK3CAIPTEN alterations are associated with advanced neoplasia. A prospective study was performed to evaluate preoperative PCF DNA testing.

Design Over 43-months, 626 PCF specimens from 595 patients were obtained by endoscopic ultrasound (EUS)-fine needle aspiration and assessed by targeted next-generation sequencing (NGS). Molecular results were correlated with EUS findings, ancillary studies and follow-up. A separate cohort of 159 PCF specimens was also evaluated for KRASIGNAS mutations by Sanger sequencing.

Results KRAS/GNAS mutations were identified in 308 (49\%) PCs, while alterations in TP53/PIK3CA/PTEN were present in $35(6 \%)$ cases. Based on 102 (17\%) patients with surgical follow-up, KRAS/GNAS mutations were detected in 56 (100\%) IPMNs and $3(30 \%)$ MCNs, and associated with $89 \%$ sensitivity and $100 \%$ specificity for a mucinous PC. In comparison, KRAS/GNAS mutations by Sanger sequencing had a $65 \%$ sensitivity and $100 \%$ specificity. By NGS, the combination of KRAS/GNAS mutations and alterations in TP53/PIK3CA/PTEN had an $89 \%$ sensitivity and $100 \%$ specificity for advanced neoplasia. Ductal dilatation, a mural nodule and malignant cytopathology had lower sensitivities (42\%, $32 \%$ and $32 \%$, respectively) and specificities (74\%, $94 \%$ and $98 \%$, respectively).

Conclusions In contrast to Sanger sequencing, preoperative NGS of PCF for KRAS/GNAS mutations is highly sensitive for IPMNs and specific for mucinous PCs. In addition, the combination of TP53/PIK3CAIPTEN alterations is a useful preoperative marker for advanced neoplasia.

\section{INTRODUCTION}

The frequent detection of a pancreatic cyst (PC) by abdominal imaging has created a diagnostic and treatment dilemma. PCs represent a broad and diverse group of lesions that range from benign to malignant entities. For example, pseudocysts and serous cystadenomas (SCAs) do not have malignant potential and can be monitored clinically, whereas mucinous PCs, such as intraductal papillary mucinous neoplasms (IPMNs) and mucinous cystic neoplasms (MCNs), can progress to invasive pancreatic ductal adenocarcinoma. ${ }^{12}$ However, distinguishing one cyst from another can be challenging on the basis of standard clinical findings, imaging parameters and ancillary fluid studies. ${ }^{3}$ Moreover, the rate of progression of mucinous PCs into malignancy is low and difficult to predict. Weighing the risks of cancer development with the risks of surgical intervention, both consensus-based and evidence-based guidelines were developed to aid in the appropriate surveillance and treatment of PCs. ${ }^{45}$ While these guidelines represent an extrapolation of current data, several studies have found them to be imperfect. ${ }^{6-8}$ Hence, the management of PCs is often an individualised approach.

Recently, DNA-based testing has emerged as an adjunct to the assessment of PCs. ${ }^{9}$ Although cellular content and fluid volume of PC aspirates are often suboptimal for routine ancillary studies, such as cytopathology and carcinoembryonic antigen (CEA) quantitation, DNA from lysed or exfoliated cyst epithelial lining shed into the pancreatic cyst fluid (PCF) can be analysed for genetic abnormalities. $^{10} 11$ Furthermore, sequencing studies have identified distinct mutational profiles of the major PCs as well as those that have progressed to invasive adenocarcinoma. ${ }^{12-14}$ For example, mutations in KRAS are commonly detected in IPMNs and MCNs and the presence of GNAS mutations is highly specific for IPMNs. ${ }^{15-17}$ In contrast, VHL mutations and/or deletions are characteristic of SCAs and CTNNB1 mutations in the absence of other genetic alterations are observed in solid-pseudopapillary neoplasms. ${ }^{13}$ Additionally, IPMNs with advanced neoplasia (high-grade dysplasia and invasive adenocarcinoma) are reported to harbour mutations in TP53, PIK3CA, PTEN and/or AKT1. ${ }^{18-23}$ While several studies have evaluated DNA testing of PCs, they have largely been retrospective in design, using postoperative specimens, limited in sample size and/ 
Significance of this study

What is already known on this subject?

- DNA-based testing has emerged as an adjunct to the assessment of pancreatic cysts (PCs).

- Although cellular content and fluid volume of PC aspirates are commonly suboptimal for routine ancillary studies, such as cytopathology and carcinoembryonic antigen (CEA) quantitation, DNA from lysed or exfoliated cyst epithelial lining shed into the PC fluid can be analysed for genetic abnormalities.

- Several studies have evaluated DNA-based testing of PCS, but they have largely been retrospective in design, using postoperative specimens, limited in sample size, lack adequate follow-up and/or suffer from insensitive detection strategies.

\section{What are the new findings?}

- We have prospectively evaluated preoperative DNA-based testing of PC fluid within a large cohort and found mutations in KRAS and/or GNAS by next-generation sequencing (NGS) are highly sensitive and specific for intraductal papillary mucinous neoplasms (IPMNs), but not mucinous cystic neoplasms (MCNs).

- The sensitivity of preoperative DNA-based testing for IPMNs and MCNs was lower with Sanger sequencing than NGS.

- The preoperative detection of mutations/deletions in TP53, PIK3CA and/or PTEN with mutant allele frequencies (MAFs) that are equivalent to MAFs for KRAS and/or GNAS mutations was highly sensitive and specific for IPMNs with advanced neoplasia (high-grade dysplasia and invasive adenocarcinoma).

- Low-level mutations in TP53, PIK3CA and/or PTEN were detected in IPMNs with low-grade dysplasia and may represent a subset of IPMNs at risk for malignant transformation.

- MAFs for GNAS mutations $>55 \%$ correlated with IPMNs with high-grade dysplasia.

\section{How might it impact on clinical practice in the foreseeable} future?

- These results definitively highlight the usage of preoperative NGS in accurately classifying PCs and the detection of IPMNs harbouring advanced neoplasia.

or lack adequate follow-up. ${ }^{12} 142425$ Thus, the diagnostic usage of DNA analysis of PCF in routine clinical practice remains unclear.

In this study, we developed a highly sensitive, targeted next-generation sequencing (NGS) assay for genes known to be frequently mutated and/or deleted in PCs and PCs with advanced neoplasia (KRAS, GNAS, NRAS, HRAS, BRAF, CTNNB1, TP53, PIK3CA, PTEN and AKT1). Due to technical issues, we were unable to include $V H L$ within this panel, but assessed the entire coding sequence of VHL by Sanger sequencing with the understanding that the sensitivity of Sanger sequencing is known to be lower than NGS. ${ }^{26}$ This test was performed within a Clinical Laboratory Improvement Amendments (CLIA)-certified and College of American Pathologists (CAP)-accredited clinical laboratory using PCF obtained by endoscopic ultrasound-fine needle aspiration (EUS-FNA) for the routine assessment of PCs. Our objectives were to prospectively evaluate DNA-based molecular testing on a large, consecutive cohort of patients to (1) identify the prevalence and distribution of genetic alterations within PCs;
(2) determine the accuracy of molecular analysis using both NGS and Sanger sequencing and (3) based on follow-up diagnostic surgical pathology compare these findings with other accepted diagnostic modalities in the preoperative assessment of PCs.

\section{MATERIALS AND METHODS \\ Cases}

Study approval was obtained from the University of Pittsburgh Institutional Review Board (IRB\# PRO13020493). Between January 2014 and July 2017, 673 PCF specimens obtained by EUS-FNA were prospectively submitted to the Molecular \& Genomic Pathology Laboratory at the University of Pittsburgh Medical Centre (UPMC) for molecular testing. In all cases, the indication for PCF molecular testing was a clinical concern for a mucinous PC. Medical records were reviewed to document patient demographics, clinical presentation, EUS findings, fluid viscosity (as noted by the endoscopist), CEA analysis and cytopathological diagnoses. Endoscopic criterion of main duct dilatation was defined by a diameter $\geq 5 \mathrm{~mm} .{ }^{4}$ In addition, the presence of a mural nodule was defined as a uniform echogenic nodule of any size without a lucent centre or hyperechoic rim. ${ }^{27}$ A value $>192 \mathrm{ng} / \mathrm{mL}$ was used as a cut-off for an elevated PCF CEA. For cytopathology specimens, specimen adequacy was assessed in all cases using a three-tiered system: satisfactory, less than optimal and unsatisfactory. Satisfactory was defined as the presence of sufficient epithelial cells and/or mucin representative of the target cyst. Less than optimal consisted of scant epithelium in the absence of mucin, but with at least few histiocytes present. Unsatisfactory specimens were virtually acellular and lacked mucin. Malignant cytopathology was defined as either at least suspicious for adenocarcinoma or positive for adenocarcinoma. Pathology slides were reviewed for each surgical specimen and diagnoses for all PCs were rendered based on standard histomorphological criteria. ${ }^{28}$ Cases diagnostic for a mucinous PC (IPMN and MCN) with high-grade dysplasia or invasive adenocarcinoma were interpreted as advanced neoplasia. ${ }^{7}$ Pathological staging was performed as outlined by the American Joint Committee on Cancer Cancer Staging Manual (eighth edition). ${ }^{29}$

\section{Molecular testing}

Molecular testing was performed prospectively as part of clinical care and within a 14-day (mean, 10 days) turnaround within the CLIA-certified and CAP-accredited Molecular and \& Genomic Pathology Laboratory at UPMC at a cost of $\$ 750$ per PCF specimen. Genomic DNA was isolated from PCF obtained by EUS-FNA using the MagNA Pure LC Total Nucleic Acid Isolation Kit (Roche, Indianapolis, Indiana, USA) on Compact MagNA Pure (Roche, Indianapolis, Indiana, USA). Extracted DNA was quantitated on the Qubit V.2.0 Fluorometer using the dsDNA HS Assay Kit (Thermo Fisher Scientific, Waltham, Maryland, USA). Amplification-based targeted NGS (PancreaSeq) was performed with primers for genomic regions of interest that included KRAS, GNAS, NRAS, HRAS, BRAF, CTNNB1, TP53, PIK3CA, PTEN and AKT1 with primer sequences and performance characteristics as previously described. ${ }^{12}{ }^{30}$ Amplicons were barcoded, purified and ligated with specific adapters. DNA quantity and quality check was performed using the 2200 TapeStation (Agilent Technologies, Santa Clara, California, USA). The Ion One Touch 2 and One Touch ES were used to prepare and enrich templates and enable testing via Ion Sphere Particles on a semiconductor chip. Massively parallel sequencing was carried out on an Ion Torrent Personal Genome Machine Sequencer or Ion Proton according to the manufacturer's instructions 
(Thermo Fisher Scientific, Waltham, Maryland, USA) and analysed with the Torrent Suite Software V.3.4.2. Bioinformatic data analysis is described further within the online supplementary material. The limit of detection was 5\% mutant allele frequency $(\mathrm{MAF})$ at $500 \times$ or $3 \% \mathrm{MAF}$ at $1000 \times$ coverage for each tested region. The minimal depth of coverage was $500 \times$. For each mutation identified, a MAF was calculated based on the number of reads of the mutant allele versus the wild-type allele and reported as a percentage. Copy number assessment was performed as previously described. ${ }^{31}$ The total depth of sequencing coverage at each sequenced region normalised by the normal controls was calculated per sequenced case. A decrease in sequencing coverage below established cut-offs with simultaneous presence of sequence variant at high MAF was considered a biallelic inactivation.

The VHL gene was sequenced by Sanger sequencing approach. For the detection of a mutation or deletion, DNA was amplified with primers flanking VHL exons 1 (5'-GCG AAG ACT ACG GAG-3' and 5'-CCG TGC TAT CGT CCC T-3'), exon 2 (5'-GTT TCA CCA CGT TAG CCA-3' and 5'-TAC AAA TAC ATC ACT TCC A-3') and exon 3 (5'-CTC TTG TTC GTT CCT TGT-3' and $5^{\prime}$-AAG CAA TGG TGC CTA TT-3'). The quality of amplified PCR product was evaluated by agarose gel electrophoresis. Then, bidirectional Sanger sequencing was performed using the BigDye Terminator Kit on ABI3730 (Thermo Fisher Scientific, Waltham, Maryland, USA). The detection of mutations was performed with Mutation Surveyor V.3.01 (SoftGenetics, State College, Pennsylvania). The limit of detection was approximately $10 \%-20 \%$ of mutant alleles present in a background of normal DNA. The aforementioned primers include individual exon-intron boundaries, and, therefore, allows for detection of both deletions and insertions within exons or complete loss of an exon by visual inspection of electropherograms. However, a limitation of this method is that it does not detect loss of the entire VHL gene.

\section{Statistical analysis}

Differences in mutational status were compared using Fisher exact test for dichotomous variables. Sensitivity and specificity were calculated using standard $2 \times 2$ contingency tables for cases with confirmed diagnostic pathology. All statistical analyses were performed using the SPSS Statistical software, V.23 (IBM, Armonk, New York, USA) and statistical significance was defined as a $\mathrm{p}$ value of $<0.05$.

\section{RESULTS}

Molecular testing and correlative clinicopathological findings

In total, 673 EUS-FNA-obtained PCF specimens from 642 patients were prospectively analysed for genetic alterations over a 43-month time period (figure 1). Among these cases, 626 (93\%) specimens from 595 patients were satisfactory for molecular testing (table 1 and online supplementary data). The remaining 47 cases were unable to be tested due to insufficient DNA for evaluation. For 6 of 595 (1\%) patients, two separate specimens corresponding to separate PCs were submitted for molecular testing. Further, 25 of 595 (4\%) patients had repeat EUS-FNA and molecular testing of their PC during the study period.

Although sufficient for molecular studies, the amount of cyst fluid was insufficient for CEA analysis in 174 of 626 (28\%) cases. In addition, 375 (60\%) specimens were either less than optimal $(n=297,47 \%)$ or unsatisfactory $(n=78,13 \%)$ for cytopathological diagnosis. The primary reason for specimen inadequacy was absent-to-scant cellularity.

The DNA concentration from submitted EUS-FNA-obtained PCF specimens ranged between 0.01 and $248 \mathrm{ng} / \mathrm{uL}$ (mean,

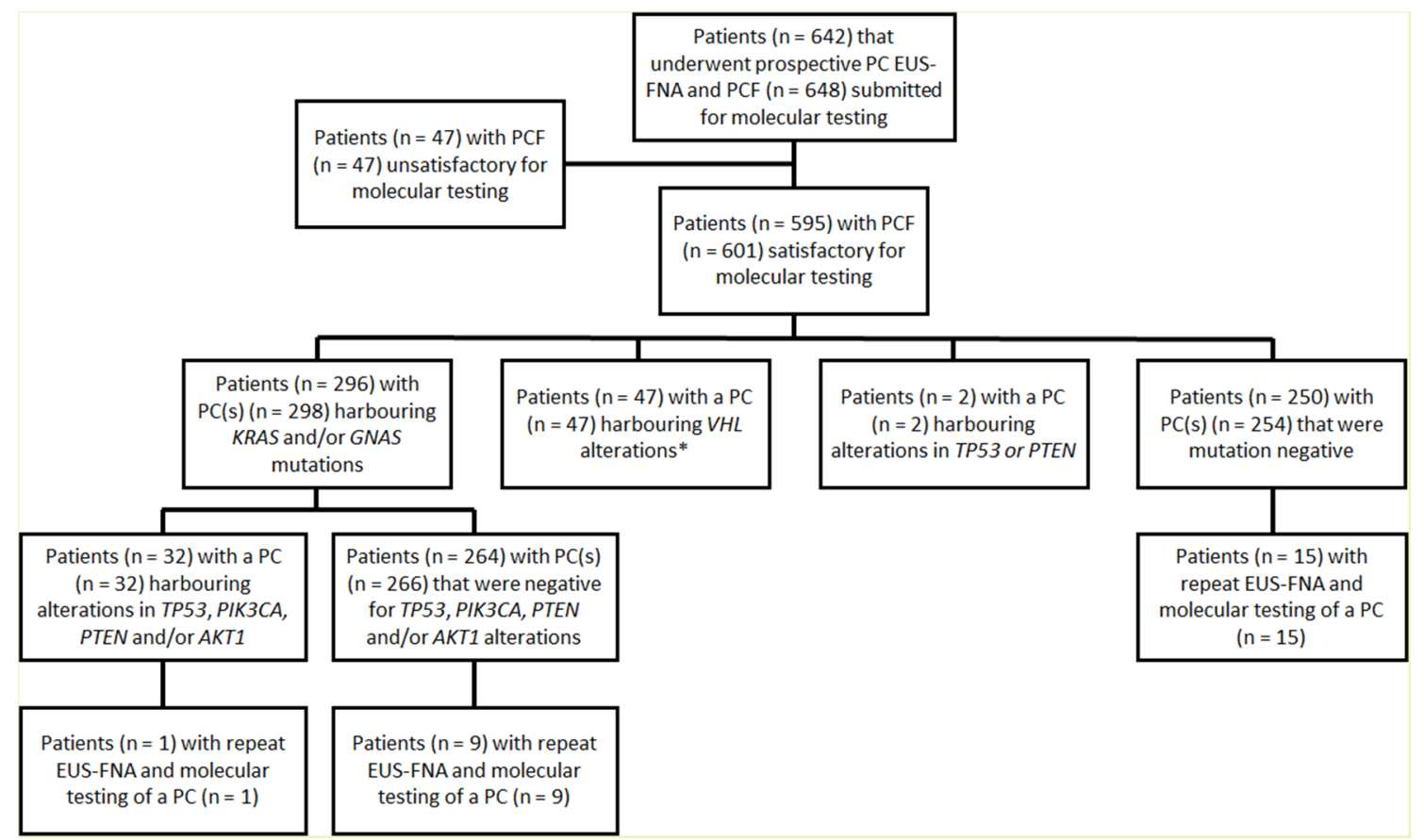

Figure 1 Study cohort. In total, 642 patients with 648 PCs underwent an EUS-FNA and PCF) molecular testing. Forty-seven PCF specimens from 47 patients were unsatisfactory for molecular testing; however, the remaining 601 PCF specimens from 595 patients were satisfactory. Repeat EUSFNA and molecular testing was performed for 25 PCs from 25 (of 595) patients. $\left(^{*}\right.$ ) Of note, 1 of 47 PCs with a VHL alteration also harboured a TP53 mutation. EUS-FNA, endoscopic ultrasound-fine needle aspiration; PC, pancreatic cyst; PCF, pancreatic cyst fluid. 
Table 1 Clinical and pathological characteristics of 595 patients with PCs and correlation with KRAS, GNAS and VHL status

\begin{tabular}{|c|c|c|c|c|c|c|c|}
\hline \multirow[b]{2}{*}{ Patient or cyst characteristics } & \multirow[b]{2}{*}{ Total } & \multicolumn{2}{|c|}{$K R A S$ and/or GNAS } & \multirow[b]{2}{*}{ p Value } & \multicolumn{2}{|c|}{ VHL } & \multirow[b]{2}{*}{ p Value } \\
\hline & & Wild type & Mutant & & Wild type & Mutant & \\
\hline Gender & $n=595$ & & & & & & \\
\hline Woman & 341 & $181(53 \%)$ & $160(47 \%)$ & 0.246 & $308(90 \%)$ & $33(10 \%)$ & 0.067 \\
\hline Man & 254 & $122(48 \%)$ & $132(52 \%)$ & & $240(94 \%)$ & $14(6 \%)$ & \\
\hline Mean age (range) (years) & $65.0(15-93)$ & $60.7(15-90)$ & $69.4(34-93)$ & $<0.001$ & $65.2(15-93)$ & $62.3(33-81)$ & 0.122 \\
\hline Symptomatic presentation & 198 & $112(57 \%)$ & $86(43 \%)$ & 0.056 & $189(95 \%)$ & $9(5 \%)$ & 0.036 \\
\hline Location & $n=626$ & & & & & & \\
\hline Head, neck and uncinate & 320 & $137(43 \%)$ & $183(57 \%)$ & $<0.001$ & $299(92 \%)$ & $21(7 \%)$ & 0.368 \\
\hline Body and tail & 306 & $181(59 \%)$ & $125(41 \%)$ & & $280(92 \%)$ & $26(8 \%)$ & \\
\hline Mean cyst size (range) (cm) & $2.7(0.8-21.0)$ & $3.0(0.8-21.0)$ & $2.4(0.8-11.0)$ & $<0.001$ & $2.7(0.8-21.0)$ & $3.2(1.0-8.9)$ & 0.060 \\
\hline Cyst multifocality & 281 & $95(34 \%)$ & $186(66 \%)$ & $<0.001$ & $270(96 \%)$ & $11(4 \%)$ & 0.002 \\
\hline Increased fluid viscosity & 319 & $85(27 \%)$ & $234(73 \%)$ & $<0.001$ & $316(99 \%)$ & $3(1 \%)$ & $<0.001$ \\
\hline CEA $>192 \mathrm{ng} / \mathrm{mL}(\mathrm{n}=452)^{*}$ & 146 & $38(26 \%)$ & $108(74 \%)$ & $<0.001$ & $146(100 \%)$ & $0(0 \%)$ & $<0.001$ \\
\hline Satisfactory cytological adequacy & 251 & $117(47 \%)$ & $134(53 \%)$ & 0.088 & $236(94 \%)$ & $15(6 \%)$ & 0.279 \\
\hline Diagnostic pathology & $n=102$ & $n=43$ & $n=59$ & & $n=100$ & $n=2$ & \\
\hline Adenocarcinoma arising in an IPMN & 13 & $0(0 \%)$ & $13(100 \%)$ & & $13(100 \%)$ & $0(0 \%)$ & \\
\hline IPMN with low-grade/high-grade dysplasia & 43 & $0(0 \%)$ & $43(100 \%)$ & $<0.001 \dagger$ & $43(100 \%)$ & $0(0 \%)$ & \\
\hline MCN with low-grade/high-grade dysplasia & 10 & $7(70 \%)$ & $3(30 \%)$ & & $10(100 \%)$ & $0(0 \%)$ & \\
\hline Serous cystadenoma & 3 & $3(100 \%)$ & $0(0 \%)$ & & $1(33 \%)$ & $2(67 \%)$ & $<0.001 \ddagger$ \\
\hline Cystic PanNET & 9 & $9(100 \%)$ & $0(0 \%)$ & & $9(100 \%)$ & $0(0 \%)$ & \\
\hline Acinar cell cystadenoma & 1 & $1(100 \%)$ & $0(0 \%)$ & & $1(100 \%)$ & $0(0 \%)$ & \\
\hline Pseudocyst & 17 & $17(100 \%)$ & $0(0 \%)$ & & $17(100 \%)$ & $0(0 \%)$ & \\
\hline Retention cyst & 2 & $2(100 \%)$ & $0(0 \%)$ & & $2(100 \%)$ & $0(0 \%)$ & \\
\hline Lymphoepithelial cyst & 2 & $2(100 \%)$ & $0(0 \%)$ & & $2(100 \%)$ & $0(0 \%)$ & \\
\hline Epidermoid cyst & 1 & $1(100 \%)$ & $0(0 \%)$ & & $1(100 \%)$ & $0(0 \%)$ & \\
\hline Squamoid cyst & 1 & $1(100 \%)$ & $0(0 \%)$ & & $1(100 \%)$ & $0(0 \%)$ & \\
\hline
\end{tabular}

*Sufficient PCF for CEA analysis was available for 127 (80\%) PCs.

tFollow-up NGS testing for KRAS and GNAS was performed for 24 (15\%) PCs.

‡p Value corresponds to SCA versus other PCs.

CEA, carcinoembryonic antigen; IPMN, intraductal papillary mucinous neoplasm; MCN, mucinous cystic neoplasm; PanNET, pancreatic neuroendocrine tumour; PC, pancreatic cyst; PCF, pancreatic cyst fluid; SCA, serous cystadenomas.

$6.93 \mathrm{ng} / \mathrm{uL}$; median, $4.7 \mathrm{ng} / \mathrm{uL})$. Overall, genetic alterations using the 11-gene panel were detected in 357 (57\%) PCs. NGS revealed activating mutations in KRAS, GNAS, BRAF and CTNNB1 in 264 (42\%), 162 (26\%), 5 (1\%) and 4 (1\%) cases, respectively. No mutations in HRAS and NRAS were detected. In total, KRAS and/or GNAS mutations were identified in 308 (49\%) PCs with $119(19 \%)$ cases harbouring mutations in both genes (online supplementary table 2). Multiple KRAS mutations were present in 10 specimens and included various combinations of codon 12,13 and 61 substitutions. MAFs for KRAS were 3\%-55\% (mean, 24\%; median, 24\%). Multiple mutations in GNAS were detected in three specimens and consisted of substitutions in codons 201 and 227. GNAS MAFs were 3\%-92\% (mean, 28\%; median, 26\%). Two PCs had a GNAS MAF of $>55 \%$. The MAFs for these two cases were $88 \%$ and $92 \%$. BRAF and CTNNB1 MAFs were $24 \%-46 \%$ and $6 \%-46 \%$, respectively. The presence of BRAF and CTNNB1 mutations were only seen in the setting of a KRAS and/or GNAS mutation. Instead of NGS, exons 1-3 of $V H L$ were evaluated by Sanger sequencing. VHL mutations and/or deletions were identified throughout the gene coding sequence in 47 of 626 (8\%) PCs.

NGS was also used to assess the status of TP53, PIK3CA, PTEN and AKT1 (table 2). Genetic alterations in TP53, PIK3CA, PTEN and AKT1 were identified in 24 (4\%), $11(2 \%), 2(1 \%)$ and $1(1 \%)$ PCs, respectively. Overall, TP53, PIK3CA, PTEN and AKT1 alterations were present in $35(6 \%)$ cases. For TP53 and PTEN, genetic alterations consisted of mutations and/or deletions throughout the gene coding sequence. Among these cases, MAFs for TP53, PTEN and AKT1 were 4\%-43\%, 11\% and $8 \%$, respectively. In addition, homozygous deletions in TP53 and PTEN were detected in $2(1 \%)$ and $1(1 \%)$ cases, respectively. Alterations in PIK3CA corresponded to activating point mutations in exon $9(\mathrm{n}=7)$ and/or exon $20(\mathrm{n}=5)$ with MAFs of 3\%-50\%.

PCs with alterations in TP53, PIK3CA and/or PTEN were associated with co-mutation(s) in KRAS and/or GNAS mutations $(\mathrm{p}<0.001)$. However, 3 of 35 PCs that had a TP53 mutation $(\mathrm{n}=2)$ or PTEN deletion $(\mathrm{n}=1)$ were wild type for KRAS and GNAS. Among the two TP53 mutant cases, one harboured a VHL deletion and the other was negative for genetic alterations. No other genetic alterations were detected in the single PC with a PTEN deletion.

\section{Follow-up information and correlation with diagnostic surgical pathology}

Follow-up data were available for 571 of 595 (96\%) patients and ranged from 1 to 42 months (mean, 27 months; median, 26 months). Diagnostic pathology was available for 102 of 595 (17\%) patients that underwent surgical resection within 1-16 months (mean, 4 months; median, 3 months) from initial EUS-FNA and molecular testing (online supplementary table 2). Except for 2 SCAs, 8 cystic pancreatic neuroendocrine tumours (PanNETs) and 14 pseudocysts, the indications for surgery of 
Table 2 Clinical and pathological characteristics of 595 patients with PCs and correlation with TP53, PIK3CA, PTEN and AKT1 status

TP53, PIK3CA, PTEN and/or AKT1

\begin{tabular}{|c|c|c|c|c|}
\hline \multirow[b]{2}{*}{ Patient or cyst characteristics } & \multirow[b]{2}{*}{ Total } & & \multirow[b]{2}{*}{ p Value } \\
\hline & & Wild type & Mutant & \\
\hline Gender & $n=595$ & & & \\
\hline Woman & 341 & $328(96 \%)$ & $13(4 \%)$ & 0.014 \\
\hline Man & 254 & $232(92 \%)$ & $22(9 \%)$ & \\
\hline Mean age (range) (years) & $65.0(15-93)$ & $64.8(15-93)$ & $68.3(45-84)$ & 0.107 \\
\hline Symptomatic presentation & 198 & $183(92 \%)$ & $15(8 \%)$ & 0.267 \\
\hline Location & $n=626$ & & & \\
\hline Head, neck and uncinate & 320 & $299(93 \%)$ & $21(7 \%)$ & 0.301 \\
\hline Body and tail & 306 & $292(95 \%)$ & $14(5 \%)$ & \\
\hline Mean cyst size (range) (cm) & $2.7(0.8-21.0)$ & $2.7(0.8-21.0)$ & $2.8(0.9-5.2)$ & 0.739 \\
\hline Cyst size $\geq 3 \mathrm{~cm}$ & 194 & $177(91 \%)$ & $17(9 \%)$ & 0.025 \\
\hline Satisfactory cytological adequacy & 251 & $233(93 \%)$ & $18(7 \%)$ & 0.213 \\
\hline Main duct dilatation & 104 & $92(88 \%)$ & $12(12 \%)$ & 0.008 \\
\hline Presence of a mural nodule & 35 & $27(77 \%)$ & $8(23 \%)$ & $<0.001$ \\
\hline Malignant cytopathology* & 10 & $3(30 \%)$ & $7(70 \%)$ & $<0.001$ \\
\hline Mutations in KRAS and/or GNAS & 308 & $276(90 \%)$ & $32(10 \%)$ & $<0.001$ \\
\hline Diagnostic pathology & $n=102$ & $n=83$ & $n=19$ & \\
\hline Adenocarcinoma arising in an IPMN & 13 & $0(0 \%)$ & $13(100 \%)$ & \\
\hline IPMN with high-grade dysplasia & 4 & $2(50 \%)$ & $2(50 \%)$ & $<0.001 \dagger$ \\
\hline MCN with high-grade dysplasia & 2 & $2(100 \%)$ & $0(0 \%)$ & \\
\hline IPMN with low-grade dysplasia & 39 & $36(92 \%)$ & $3(8 \%)$ & \\
\hline MCN with low-grade dysplasia & 8 & $7(87 \%)$ & $1(13 \%)$ & \\
\hline Serous cystadenoma & 3 & $3(100 \%)$ & $0(0 \%)$ & \\
\hline Cystic PanNET & 9 & $9(100 \%)$ & $0(0 \%)$ & \\
\hline Non-neoplastic cysts & 24 & $24(100 \%)$ & $0(0 \%)$ & \\
\hline
\end{tabular}

* Malignant cytopathology is defined as at least suspicious for adenocarcinoma. tp Value corresponds to mucinous PCs with advanced neoplasia versus other PCs.

CEA, carcinoembryonic antigen; IPMN, intraductal papillary mucinous neoplasm; MCN, mucinous cystic neoplasm; PanNET, pancreatic neuroendocrine tumour; PC, pancreatic cyst.

the remaining PCs were due to concern for advanced neoplasia within a mucinous PC on the basis of the Fukuoka guidelines and consideration of molecular testing. ${ }^{7}$ Mutations in KRAS and/or GNAS were preoperatively detected in all 56 IPMNs. In addition, KRAS mutations were identified in two MCNs with high-grade dysplasia and one MCN with low-grade dysplasia. However, the remaining seven MCNs with low-grade dysplasia were KRAS/ GNAS-negative. The MAFs for KRAS and GNAS were 3\%-47\% and 3\%-92\%, respectively. As previously described, two PCs had MAFs of $>55 \%$, and both cases corresponded to IPMNs with high-grade dysplasia. No mutations in KRAS and/or GNAS were found in the non-mucinous PCs within the resection cohort.

$V H L$ alterations were preoperatively seen in two of three SCAs. Although Sanger sequencing failed to detect a VHL alteration in one SCA by EUS-FNA, repeat testing of the corresponding surgical resection specimen identified the presence of a VHL frameshift mutation. No alterations in VHL were observed in the remaining mucinous and non-mucinous PCs.

Genetic alterations in TP53, PIK3CA and/or PTEN were identified in all 13 IPMNs with adenocarcinoma, 2 IPMNs with highgrade dysplasia, 3 IPMNs with low-grade dysplasia and $1 \mathrm{MCN}$ with low-grade dysplasia (table 3 and online supplementary table 3) The MAFs for TP53, PIK3CA and PTEN were 8\%-43\%, $3 \%-50 \%$ and $10 \%$, respectively. Except for the one MCN with low-grade dysplasia, co-mutations in KRAS and/or GNAS were detected in all PCs with alterations in TP53, PIK3CA and/or PTEN. Among the 13 IPMNs with adenocarcinoma and 2 IPMN with high-grade dysplasia, the MAFs for KRAS and/or GNAS were at least equal to MAFs for TP53, PIK3CA and/or PTEN (figure 2). The three IPMNs with low-grade dysplasia had activating mutations in PIK3CA, but the MAFs for PIK3CA were less than the MAFs for KRAS. While no genetic alterations in TP53, PIK3CA and/or PTEN were detected in two IPMNs with highgrade dysplasia, the MAF for GNAS in both cases was $>55 \%$. The remaining 2 MCNs with high-grade dysplasia, 36 IPMNs with low-grade dysplasia, $8 \mathrm{MCNs}$ with low-grade dysplasia and non-mucinous PCs were negative for TP53, PIK3CA, PTEN and/ or AKT1 alterations.

Among the remaining 469 patients with follow-up data and no diagnostic surgical pathology, 230 (49\%) had PCs with KRAS and/or GNAS mutations. Fourteen of these 230 (6\%) patients also had mutations in TP53, PIK3CA and/or AKT1. However, the MAFs for TP53, PIK3CA and/or AKT1 (4\%-9\%) were less than MAFs for KRAS and/or GNAS (28\%-45\%). In addition, two patients harboured a PC with a TP53 mutation and MAFs of $5 \%$, but wild type for both KRAS and GNAS. One of these two TP53 mutant cases also harboured a VHL deletion. None of these PCs demonstrated concerning features for advanced neoplasia by both imaging (eg, ductal dilatation or the presence of a mural nodule) and cytopathology (eg, malignant cytopathology). Moreover, all 16 patients are currently alive and well and have not developed pancreatic cancer on follow-up.

As discussed previously, 25 of 595 patients had repeat EUS-FNA and molecular testing of their PC (figure 1). Fifteen of 25 patients harboured a PC with no detectable alterations, while the remaining 10 patients had a KRAS and/or GNAS mutant PC. Repeat aspiration and molecular testing of all 25 cases continued to identify the same KRAS and GNAS genetic status as initial 


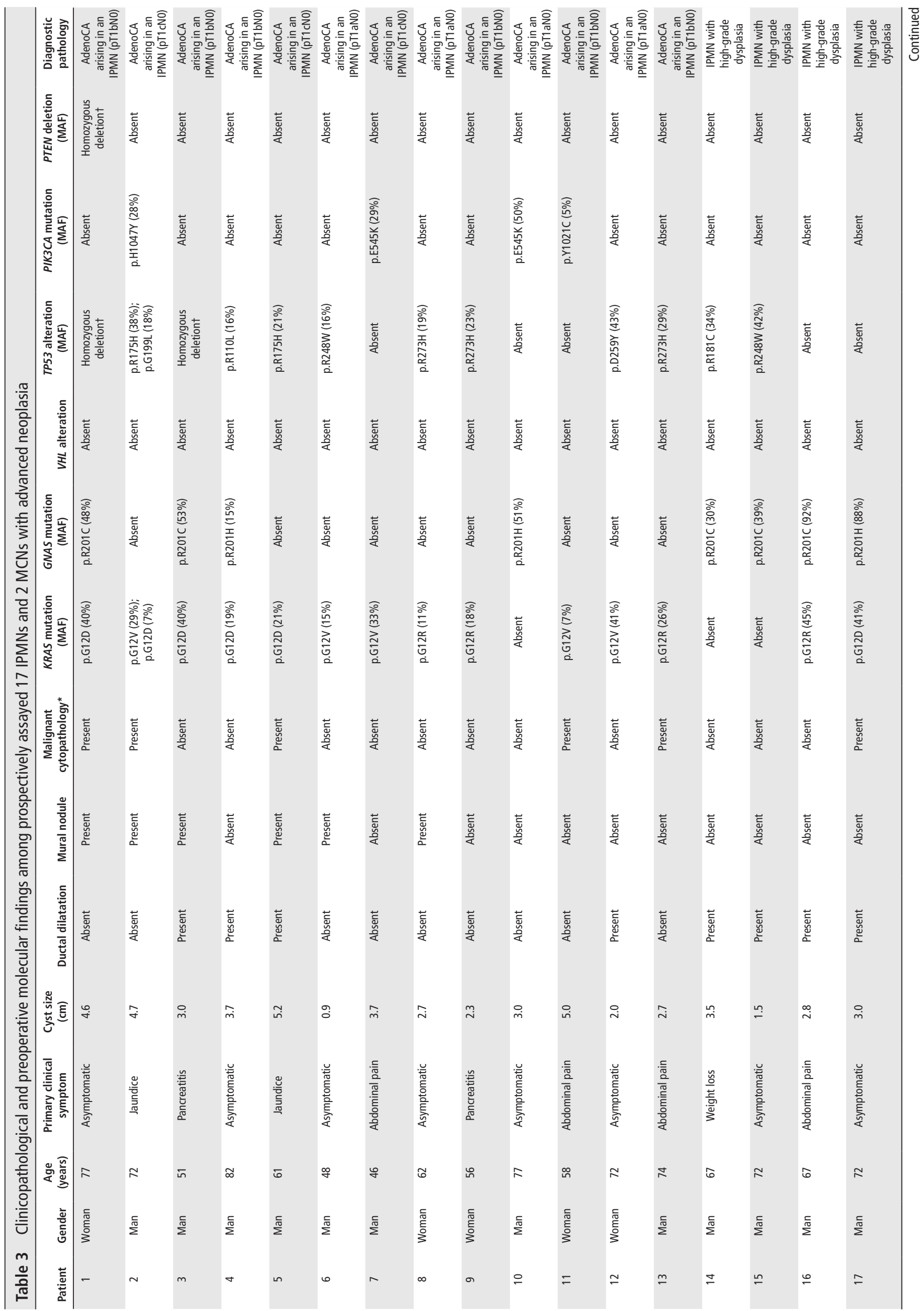


testing. However, among the $10 \mathrm{KRAS}$ and/or GNAS-mutant PCs, 1 case had a TP53 mutation on initial molecular testing that was absent on subsequent testing. For this single case, the MAFs for KRAS and TP53 were 39\% and 4\%, respectively. In contrast, the subsequent specimen had a KRAS MAF of only 4\%. A comparison of both the initial and repeat EUS-FNA specimens for the remaining six PCs revealed MAFs for KRAS and/or GNAS that were essentially the same.

\section{Comparison and combination of molecular testing with other diagnostic modalities}

Based on 102 cases with follow-up diagnostic pathology, preoperative NGS detection of KRAS and/or GNAS mutations had $100 \%$ sensitivity and 96\% specificity for an IPMN (table 4). Further, mutations in KRAS and/or GNAS mutations had a sensitivity of $89 \%$ and a specificity of $100 \%$ for both IPMNs and MCNs. Increased fluid viscosity and an elevated CEA had lower sensitivities (77\% and 57\%, respectively) and lower specificities (89\% and $80 \%$, respectively).

In conjunction with KRAS and/or GNAS mutations, alterations in TP53, PIK3CA and/or PTEN had 88\% sensitivity and 97\% specificity for IPMNs with advanced neoplasia. Both the sensitivity and specificity increased to $100 \%$ by modifying the selection criteria to include cases with either GNAS MAFs $>55 \%$ or TP53/PIK3CA/PTEN MAFs that were at least equivalent to $K R A S / G N A S$ MAFs. In comparison, main pancreatic duct dilatation and the presence of a mural nodule on EUS had sensitivities of $47 \%$ and $35 \%$, respectively, and specificities of $74 \%$ and $94 \%$, respectively. A preoperative cytopathological diagnosis of at least suspicious for adenocarcinoma was associated with 35\% sensitivity and $97 \%$ specificity.

Overall, the sensitivity and specificity of combining KRAS and/ or GNAS mutations with the presence of TP53, PIK3CA and/or PTEN alterations for a mucinous PC with advanced neoplasia was $79 \%$ and $96 \%$, respectively. Modification of selection criteria to include the detection of GNAS mutations with MAFs of $>55 \%$ or the MAFs for TP53, PIK3CA, and/or PTEN alterations of either equal or greater to MAFs for KRAS and/or GNAS mutations had $89 \%$ sensitivity and 100\% specificity. A diagnosis of at least suspicious for adenocarcinoma on cytopathology had $32 \%$ sensitivity and $98 \%$ specificity.

\section{Prospective analysis of KRAS and GNAS testing using Sanger} sequencing

Prior to this study, prospective EUS-FNA PCF testing of KRAS and GNAS was performed by Sanger sequencing for 175 PCs from 169 patients over a 12-month time period (online supplementary material and supplementary table 3). Among the 175 PCs, 159 (91\%) PCs from 153 patients were satisfactory for molecular analysis. In contrast to a prevalence of $49 \%$ by NGS, Sanger sequencing detected mutations in KRAS and/or GNAS in $39 \%$ of PCs (62 of 159). Among this cohort of 159 PCs, 34 cases had diagnostic pathology and included the following mucinous PCs: 5 IPMNs with adenocarcinoma, 1 IPMN with high-grade dysplasia, 12 IPMNs with low-grade dysplasia and 2 MCNs with low-grade dysplasia. By Sanger sequencing, 13 of 18 (72\%) IPMNs and 0 of $2(0 \%)$ MCNs were found to harbour mutations in KRAS and/or GNAS. Overall, the presence of KRAS and/ or GNAS mutations by Sanger sequencing had a sensitivity and specificity of $72 \%$ and $100 \%$, respectively, for IPMNs and $65 \%$ and $100 \%$, respectively, for both IPMNs and MCNs. Among the 159 PCs tested, 24 cases (8 KRAS/GNAS mutant and 16 KRAS/ GNAS wild type) underwent repeat testing for KRAS and GNAS 


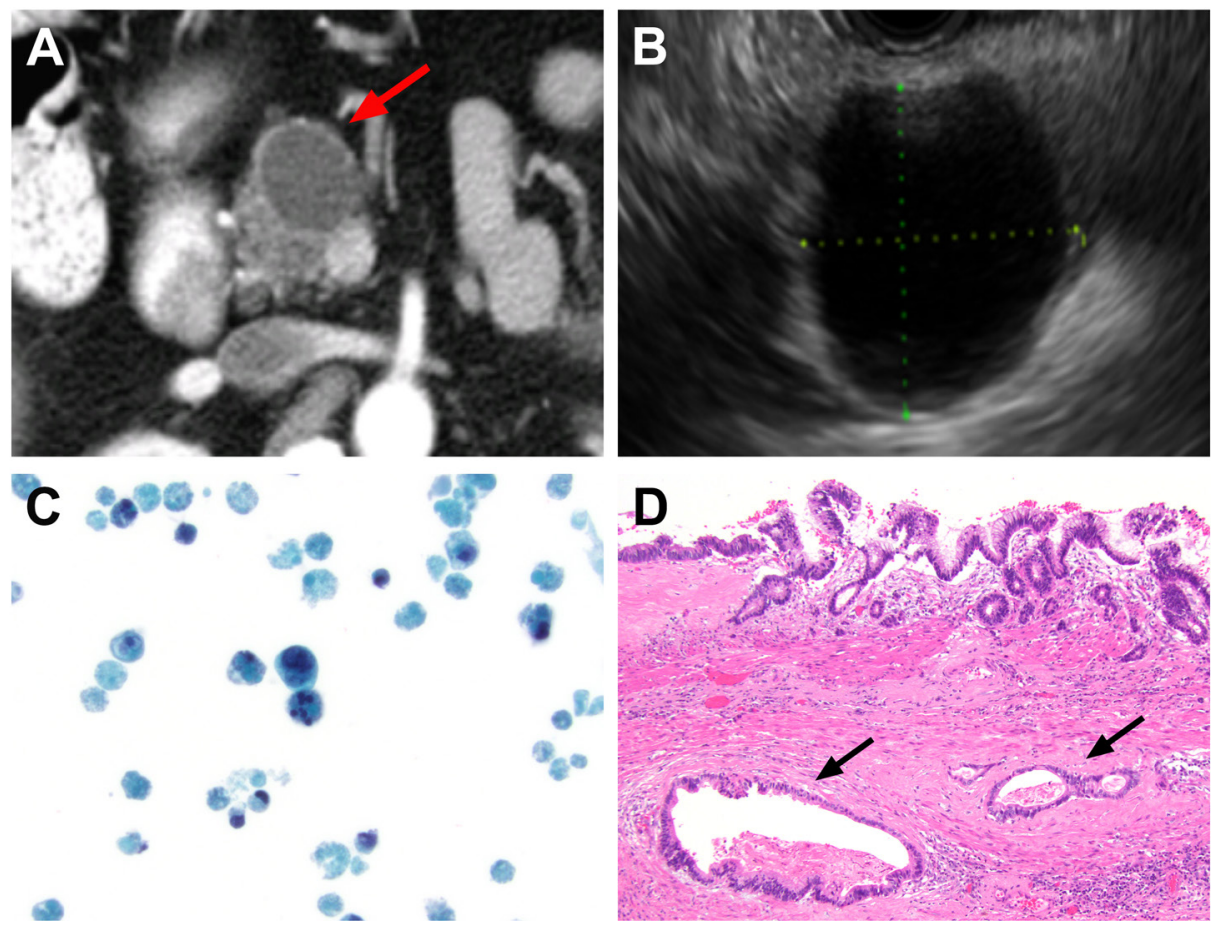

Figure 2 DNA-based molecular testing of a pancreatic head cyst. An incidental $3 \mathrm{~cm}$ pancreatic head cyst with no associated main duct dilatation or mural nodule by both (A) CT (red arrow) and (B) endoscopic ultrasound. Fine-needle aspiration and subsequent (C) cytopathology showed atypical cells with no definitive mucin. However, DNA analysis identified mutations in GNAS and PIK3CA with MAFs of 51\% and 50\%, respectively. Followup surgical resection revealed an (D) invasive moderately differentiated adenocarcinoma (black arrows) arising in an intraductal papillary mucinous neoplasm. MAFs, mutant allele frequencies.

by NGS. The status of KRAS and GNAS were essentially the same by NGS; however, 3 of 16 KRAS/GNAS wild type PCs by Sanger sequencing were found to harbour mutations in $\operatorname{KRAS}(\mathrm{n}=3)$ and/or GNAS $(\mathrm{n}=1)$ by NGS.

\section{DISCUSSION}

Although several factors should be considered when evaluating a patient with a PC, key questions need to be answered before continuing further surveillance and treatment. First, what type of PC does the patient have? More specifically, given the malignant potential of mucinous PCs, is the cyst mucinous or non-mucinous? Second, does the mucinous PC harbour malignancy? And, lastly, if not, what is the malignant potential of the mucinous PC within the patient's lifetime?

Similar to previous studies using retrospective cohorts and postsurgical specimens, our prospective evaluation of preoperative DNA-based PC testing identified mutations in KRAS and/ or GNAS to be $89 \%$ sensitive and $100 \%$ specific for a mucinous PC. Furthermore, the presence of KRAS and/or GNAS mutations reached $100 \%$ sensitivity for IPMNs, and the presence of GNAS mutations was $100 \%$ specific for an IPMN. However, KRAS mutations were detected in only $30 \%$ of MCNs. While mutations in KRAS are common in MCNs, the prevalence of these activating mutations is reported to increase with the severity of dysplasia. ${ }^{16} 17$ Jimenez et al identified KRAS mutations in $26 \%$ of MCNs with low-grade dysplasia and $89 \%$ of MCNs with highgrade dysplasia. ${ }^{32}$ Among the MCNs within our study cohort, KRAS mutations were found in $100 \%$ of MCNs with highgrade dysplasia and 13\% of MCNs with low-grade dysplasia.
Considering the relatively young age of most patients, frequent occurrence within the pancreatic body and tail and unknown risk of progression to adenocarcinoma, surgical resection is typically recommended for patients with MCNs. ${ }^{4}$ Therefore, the assessment of KRAS alone is inadequate for the detection of MCNs and additional markers are needed to improve the sensitivity of DNA testing. Of note, one MCN with low-grade dysplasia did harbour a PTEN deletion and, in the absence of a KRAS mutation, may represent a marker for MCNs. Despite the lack of sensitivity for MCNs, the sensitivity and specificity of DNA testing for a mucinous PC were higher than surrogate markers of mucinous differentiation, such as increased fluid viscosity and elevated CEA.

The diagnosis of a mucinous PC is further enhanced by molecular markers to exclude common mimics. Oligocystic and unilocular variants of SCA are often clinically and radiographically indistinguishable from branch duct IPMNs and MCNs. Genetic alterations in VHL have been described to be highly specific for SCAs, but could potentially be present in cystic PanNETs. ${ }^{33}$ Within our study, the specificity of VHL mutations and/or deletions by Sanger sequencing for SCAs was 100\%. However, we failed to preoperatively identify a VHL alteration in one SCA. Repeat testing of the corresponding surgical pathology specimen revealed a frameshift mutation in VHL. The inability to detect a VHL alteration within the preoperative PCF may be attributed to the inherent limitations of Sanger sequencing. Prior to this study, we prospectively evaluated KRAS and GNAS mutations by Sanger sequencing. In contrast to NGS, Sanger sequencing detected KRAS and GNAS mutations in 39\% of PCs and had 
Table 4 Sensitivities and specificities of molecular testing and other diagnostic modalities based on 102 surgically resected PCs

\begin{tabular}{|c|c|c|}
\hline Parameter & Sensitivity $(95 \% \mathrm{Cl})$ & Specificity $(95 \% \mathrm{Cl})$ \\
\hline \multicolumn{3}{|l|}{ IPMNs } \\
\hline KRAS and/or GNAS mutations & $100 \%(0.92$ to 1.00$)$ & $96 \%$ (0.84 to 0.99$)$ \\
\hline Presence of multiple cysts & $54 \%$ (0.40 to 0.67$)$ & $72 \%$ (0.56 to 0.84$)$ \\
\hline Increased fluid viscosity & $82 \%(0.69$ to 0.91$)$ & $80 \%(0.66$ to 0.90$)$ \\
\hline Elevated CEA* & $57 \%(0.40$ to 0.73$)$ & $70 \%(0.53$ to 0.83$)$ \\
\hline \multicolumn{3}{|l|}{ IPMNs with advanced neoplasia } \\
\hline TP53, PIK3CA and/or PTEN alterations & $88 \%(0.62$ to 0.98$)$ & $95 \%$ (0.88 to 0.98$)$ \\
\hline KRAS and/or GNAS mutations with TP53, PIK3CA and/or PTEN alterations & $88 \%(0.62$ to 0.98$)$ & $97 \%(0.89$ to 0.99$)$ \\
\hline GNAS MAF $>55 \%$ or TP53/PIK3CAIPTEN MAFs at least equal to KRAS/GNAS MAFs & $100 \%(0.77$ to 1.00$)$ & $100 \%(0.95$ to 1.00$)$ \\
\hline Main pancreatic duct dilatation & $47 \%(0.24$ to 0.71$)$ & $74 \%$ (0.63 to 0.83$)$ \\
\hline Presence of a mural nodule & $35 \%(0.15$ to 0.61$)$ & $94 \%$ (0.86 to 0.98$)$ \\
\hline Malignant cytopathology $\dagger$ & $35 \%(0.15$ to 0.61$)$ & $97 \%(0.91$ to 1.00$)$ \\
\hline \multicolumn{3}{|l|}{ IPMNs and MCNs } \\
\hline KRAS and/or GNAS mutations & $89 \%(0.79$ to 0.95$)$ & $100 \%(0.88$ to 1.00$)$ \\
\hline Increased fluid viscosity & $77 \%(0.65$ to 0.86$)$ & $89 \%(0.73$ to 0.96$)$ \\
\hline Elevated CEA* & $57 \%(0.42$ to 0.71$)$ & $80 \%(0.61$ to 0.92$)$ \\
\hline \multicolumn{3}{|l|}{ IPMNs and MCNs with advanced neoplasia } \\
\hline TP53, PIK3CA and/or PTEN alterations & $79 \%(0.54$ to 0.93$)$ & $95 \%(0.88$ to 0.98$)$ \\
\hline KRAS and/or GNAS mutations with TP53, PIK3CA and/or PTEN alterations & $79 \%(0.54$ to 0.93$)$ & $96 \%(0.89$ to 0.99$)$ \\
\hline GNAS MAF $>55 \%$ or TP53/PIK3CAIPTEN MAFs at least equal to KRAS/GNAS MAFs & $89 \%(0.66$ to 0.98$)$ & $100 \%(0.95$ to 1.00$)$ \\
\hline Main pancreatic duct dilatation & $42 \%(0.21$ to 0.66$)$ & $74 \%$ (0.63 to 0.82$)$ \\
\hline Presence of a mural nodule & $32 \%(0.14$ to 0.57$)$ & $94 \%$ (0.86 to 0.98$)$ \\
\hline Malignant cytopathology $\dagger$ & $32 \%(0.13$ to 0.57$)$ & $98 \%(0.91$ to 1.00$)$ \\
\hline
\end{tabular}

* On the basis of cases in which sufficient fluid was available for CEA testing.

tMalignant cytopathology was defined as at least suspicious for adenocarcinoma.

CEA, carcinoembryonic antigen; MAF, mutant allele frequency; PC, pancreatic cyst.

a sensitivity of $72 \%$ for IPMNs. The differences in sensitivity between Sanger sequencing and NGS can be explained by the detection limit for each assay. The lowest limit of detection for Sanger sequencing is approximately $10 \%-20 \%$ of mutant alleles, while NGS as described herein is approximately $3 \%-5 \%$ of mutant alleles. Within this study, 24\% of KRAS mutant cysts and $22 \%$ of GNAS mutant cysts had MAFs of $<10 \%$. These findings would suggest that Sanger sequencing is insufficient for preoperative VHL testing and the prevalence of VHL mutations and/ or deletions within our study cohort is likely to be inaccurate. Thus, we would discourage the use of Sanger sequencing when evaluating preoperative PCF for molecular alterations.

In addition to accurate cyst classification, PC DNA testing has garnered significant interest due to the genetic differences between mucinous PCs with low-grade dysplasia and those with high-grade dysplasia and invasive adenocarcinoma (advanced neoplasia). Alterations in the gene TP53 and those within the mTOR pathway have been implicated in the malignant transformation of mucinous PCs. The combination of KRAS and/or GNAS mutations with TP53, PIK3CA and/or PTEN alterations had 79\% sensitivity and $96 \%$ specificity for a mucinous PC with advanced neoplasia. Rosenbaum et al described their experience with preoperative NGS testing of 113 PCs with corresponding diagnostic pathology available for 38 cases. $^{25}$ The authors reported that DNA mutational analysis was associated with $46 \%$ sensitivity and $100 \%$ specificity for advanced neoplasia. Although the authors did not include PIK3CA and PTEN within their testing panel, TP53 was assessed, but only mutated in $17 \%$ of cases with advanced neoplasia. Herein, we found that $63 \%$ of mucinous PCs with advanced neoplasia harboured TP53 alterations. While both studies used NGS, the depth of coverage for each assay was significantly different. Coverage refers to the number of times a region is sequenced. The deeper the coverage of a genetic target (eg, the more times a region is sequenced), the greater the reliability and sensitivity of the sequencing assay. Within our study, the minimum depth of coverage for each genetic target was $500 \times$. In fact, we routinely achieved over $1000 \times$ depth of coverage. Rosenbaum et al aimed for a minimum depth of coverage of $100 \times$ with a median of $200 \times$ per target. ${ }^{24} 25$ Nevertheless, the authors state that MAFs as low as $5 \%$ were achieved for individual genes.

Reviewing the results of NGS testing from the entire study cohort revealed two findings that would improve the sensitivity and specificity for detecting mucinous PCs with advanced neoplasia. First, a MAF $>55 \%$ in GNAS was identified in two PCs and corresponded to IPMNs with high-grade dysplasia. Activating mutations in KRAS and GNAS are typically heterozygous and the MAF is $\leq 50 \%$ due to masking of mutations by contaminating non-neoplastic cells (eg, chronic inflammation and gastrointestinal contamination). In rare instances, the MAF of KRAS mutations is $>50 \%$ through either deletion of the wild-type allele or copy number gain of the mutant allele. This phenomenon is known as mutant allele-specific imbalance (MASI) and reflects increased dosage of the mutant allele by copy-neutral loss of heterozygosity or gene amplification. We previously reported KRAS MASI in PCF correlates with mucinous PCs with advanced neoplasia. ${ }^{11}$ To date, GNAS MASI within PCF has not been described, but based on our findings it seems to be associated with high-grade dysplasia within IPMNs.

A second finding was a MAF for TP53/PIK3CA/PTEN that was at least equal to a MAF for KRAS/GNAS correlated with the presence of advanced neoplasia within an IPMN. Although the combination of KRAS and/or GNAS mutations with genetic alterations in TP53, PIK3CA and/or PTEN were frequently 
detected in IPMNs with advanced neoplasia, mutations in KRAS/GNAS and PIK3CA were also identified in two IPMNs with low-grade dysplasia. Moreover, 10 PCs without diagnostic surgical pathology were found to harbour mutations in KRAS/ GNAS and TP53/PIK3CA/PTEN, but lacked concerning features for advanced neoplasia by both imaging and cytopathology, and have not progressed to malignancy on follow-up. However, among these cases, the MAFs for TP53/PIK3CA/PTEN were lower than the MAFs for KRAS/GNAS. By modifying the selection criteria of NGS testing to include a MAF $>55 \%$ for GNAS and a MAF for TP53/PIK3CA/PTEN that is at least equivalent to an MAF for KRAS/GNAS, the sensitivity and specificity for a mucinous PC with advanced neoplasia was $89 \%$ and $100 \%$, respectively. In comparison, a cytopathological diagnosis of at least suspicious for adenocarcinoma within a mucinous PC was associated with $32 \%$ sensitivity and $98 \%$ specificity. Thus, NGS testing seems to outperform other diagnostic modalities in the detection of a mucinous PC with high-grade dysplasia and invasive adenocarcinoma.

The presence of TP53, PIK3CA and/or PTEN alterations within IPMNs with low-grade dysplasia and IPMNs are clinically not worrisome is an intriguing observation. The traditional thought is mutations in these genes emerge as IPMNs progress from low-grade dysplasia to high-grade dysplasia and invasive adenocarcinoma. ${ }^{1}$ Recently, Yu et al described their experience with NGS of secretin-stimulated pancreatic juice samples. ${ }^{22}$ Analogous to our findings, the authors reported that TP53 mutations were detected in samples from patients with invasive adenocarcinoma and a minority of patients with low-grade IPMNs. In addition, higher concentrations of mutant TP53 were present in cases with invasive adenocarcinomas as compared with IPMNs with low-grade dysplasia. The authors also described a case where a TP53 mutation was identified 1 year prior to the diagnosis of invasive adenocarcinoma and at a time when no worrisome features were evident by imaging. While the natural history of IPMNs with low-level alterations in TP53, PIK3CA and/or PTEN is relatively unknown, it is plausible that these IPMNs are at a high risk for progression to malignancy. Considering $49 \%$ of PCs within our study cohort are likely mucinous PCs with majority representing IPMNs, a rational and cost-effective strategy for PC surveillance is needed. The identification of KRAS and/or GNAS mutations and low-level alterations in TP53, PIK3CA and/or PTEN in PCF may represent a predictive marker of malignant potential within an IPMN.

This study is, however, not without limitations. Although a large number of PCs were analysed, diagnostic surgical pathology was available for a small proportion of patients that represent a surgical selection bias. Our study also suffers from a testing selection bias as PC specimens satisfactory for NGS and Sanger sequencing were used for analysis. Considering $7 \%$ of cases failed molecular testing, the effect of this selection bias on our results is likely to be minimal. In addition, the follow-up period of this study is relatively short to assess the clinical impact of detecting alterations in TP53, PIK3CA, PTEN and/or AKT1. The results published herein represent our initial findings and we plan to continue monitoring these patients over their lifetime. Further, the molecular panel consisted of 11 genes commonly altered in PCs and present in invasive adenocarcinoma, but did not include RNF43, CDKN2A and SMAD4. Mutations and/or deletions in RNF43 have been identified in both IPMNs and MCNs, and may improve the sensitivity of detecting MCNs. ${ }^{13}$ However, RNF43 alterations have a prevalence of $8 \%$ to $35 \%$ in MCNs and, therefore, the addition of RNF43 may have limited usage in PC testing. ${ }^{22}{ }^{34}$ Deletions in CDKN2A and SMAD4 are associated with IPMNs harbouring high-grade dysplasia and invasive adenocarcinoma. ${ }^{182225}$ While these genes may improve the accuracy of detecting molecular testing, using the aforementioned selection criteria to include MAFs for GNAS and TP53/PIK3CA/PTEN alterations, the sensitivity and specificity for advanced neoplasia in an IPMN was $100 \%$, respectively. Nevertheless, additional studies are required to identify a minimal set of genes necessary for the assessment of PCs. Lastly, this study does not address the optimal approach of integrating DNA-based molecular testing to current PC surveillance protocols. Previously, we proposed an algorithmic approach to the management of PCs by utilizing molecular testing to stratify patients for appropriate management, but this requires further validation before implementation. ${ }^{7}$ Herein, the Fukuoka guidelines were applied to the evaluation of mucinous PCs with consideration of the impact of molecular testing based on prior studies. ${ }^{712-14} 1619-2325$

In summary, we report the results of a large, prospective study of DNA-based molecular testing of EUS-FNA-obtained preoperative PCF. Overall, our results support the usage of NGS analysis in PCF given the high sensitivity and specificity in classifying PCs, especially IPMNs, and in the diagnosis of IPMNs with advanced neoplasia. Notable limitations of DNA-based molecular testing include the assessment of MCNs using KRAS and usage of Sanger sequencing in the evaluation of PCs. Future studies are required to explore the integration of DNA-based molecular testing into current management guidelines.

Acknowledgements The authors thank the clinical coordinating staff (Tracy Hoteck, Becky Pottinger, Laura A Osman, Nicole R Habel, Leslie Minteer and Kimberly N Stewart) at the UPMC Digestive Disorders Center. In addition, they thank Robyn $\mathrm{L}$ Roche and Kate Smith for outstanding administrative assistance. Lastly, this study would not be possible without the support of the Pancreatic Cancer Action Network, Pittsburgh Affiliate, and the National Pancreas Foundation, Western Pennsylvania Chapter.

Contributors ADS, KM, REB, AK, HJZ, AS, AIW and MNN: study concept and design. ADS, KG, REB, AK, HJZ, JSC, KEF, GIP, AS, DLB, AKD, MH, KKL, JWM, SEM, NPO, JFP, AT, AHZ, AIW and MNN: acquisition of data. ADS, AIW and MNN: analysis and interpretation of data. ADS, KM, HJZ and MNN: drafting of the manuscript.

Funding This study was supported in part by grants from the Pancreatic Cancer Action Network (Translational Research Grant), National Pancreas Foundation, Western Pennsylvania Chapter and the University of Pittsburgh (to AD Singhi).

Competing interests None declared.

\section{Patient consent Obtained.}

Ethics approval University of Pittsburgh Institutional Review Board.

Provenance and peer review Not commissioned; externally peer reviewed.

Open access This is an open access article distributed in accordance with the Creative Commons Attribution Non Commercial (CC BY-NC 4.0) license, which permits others to distribute, remix, adapt, build upon this work non-commercially, and license their derivative works on different terms, provided the original work is properly cited and the use is non-commercial. See: http://creativecommons.org/ licenses/by-nc/4.0/

(c) Article author(s) (or their employer(s) unless otherwise stated in the text of the article) 2018. All rights reserved. No commercial use is permitted unless otherwise expressly granted.

\section{REFERENCES}

1 Matthaei H, Schulick RD, Hruban RH, et al. Cystic precursors to invasive pancreatic cancer. Nat Rev Gastroenterol Hepatol 2011;8:141-50.

2 Hruban RH, Maitra A, Kern SE, et al. Precursors to pancreatic cancer. Gastroenterol Clin North Am 2007;36:831-49.

3 Thiruvengadam N, Park WG. Systematic review of pancreatic cyst fluid biomarkers: the path forward. Clin Trans/ Gastroenterol 2015;6:e88

4 Tanaka M, Fernández-del Castillo C, Adsay V, et al. International consensus guidelines 2012 for the management of IPMN and MCN of the pancreas. Pancreatology 2012;12:183-97. 
5 Vege SS, Ziring B, Jain R, et al. American gastroenterological association institute guideline on the diagnosis and management of asymptomatic neoplastic pancreatic cysts. Gastroenterology 2015;148:819-22.

6 Goh BK, Lin Z, Tan DM, et al. Evaluation of the fukuoka consensus guidelines for intraductal papillary mucinous neoplasms of the pancreas: Results from a systematic review of 1,382 surgically resected patients. Surgery 2015;158:1192-202.

7 Singhi AD, Zeh HJ, Brand RE, et al. American gastroenterological association guidelines are inaccurate in detecting pancreatic cysts with advanced neoplasia: a clinicopathologic study of 225 patients with supporting molecular data. Gastrointest Endosc 2016:83:1107-17.

8 Kaimakliotis P, Riff B, Pourmand K, et al. Sendai and fukuoka consensus guidelines identify advanced neoplasia in patients with suspected mucinous cystic neoplasms of the pancreas. Clin Gastroenterol Hepatol 2015;13:1808-15.

9 Theisen BK, Wald Al, Singhi AD. Molecular diagnostics in the evaluation of pancreatic cysts. Surg Pathol Clin 2016;9:441-56.

10 Khalid A, McGrath KM, Zahid M, et al. The role of pancreatic cyst fluid molecular analysis in predicting cyst pathology. Clin Gastroenterol Hepatol 2005;3:967-73.

11 Khalid A, Zahid M, Finkelstein SD, et al. Pancreatic cyst fluid DNA analysis in evaluating pancreatic cysts: a report of the PANDA study. Gastrointest Endosc 2009;69:1095-102.

12 Springer S, Wang Y, Dal Molin M, et al. A combination of molecular markers and clinical features improve the classification of pancreatic cysts. Gastroenterology 2015;149:1501-10.

13 Wu J, Jiao Y, Dal Molin M, et al. Whole-exome sequencing of neoplastic cysts of the pancreas reveals recurrent mutations in components of ubiquitin-dependent pathways. Proc Nat/ Acad Sci U S A 2011;108:21188-93.

14 Amato E, Molin MD, Mafficini A, et al. Targeted next-generation sequencing of cancer genes dissects the molecular profiles of intraductal papillary neoplasms of the pancreas. J Pathol 2014;233:217-27.

15 Singhi AD, Nikiforova MN, Fasanella KE, et al. Preoperative GNAS and KRAS testing in the diagnosis of pancreatic mucinous cysts. Clin Cancer Res 2014;20:4381-9.

16 Wu J, Matthaei H, Maitra A, et al. Recurrent GNAS mutations define an unexpected pathway for pancreatic cyst development. Sci Trans/ Med 2011;3:92ra66.

17 Nikiforova MN, Khalid A, Fasanella KE, et al. Integration of KRAS testing in the diagnosis of pancreatic cystic lesions: a clinical experience of 618 pancreatic cysts. Mod Pathol 2013;26:1478-87.

18 Pea A, Yu J, Rezaee N, et al. Targeted DNA sequencing reveals patterns of local progression in the pancreatic remnant following resection of intraductal papillary mucinous neoplasm (IPMN) of the pancreas. Ann Surg 2017;266:133-41.
19 Kanda M, Sadakari Y, Borges M, et al. Mutant TP53 in duodenal samples of pancreatic juice from patients with pancreatic cancer or high-grade dysplasia. Clin Gastroenterol Hepatol 2013;11:719-30

20 Schönleben F, Qiu W, Ciau NT, et al. PIK3CA mutations in intraductal papillary mucinous neoplasm/carcinoma of the pancreas. Clin Cancer Res 2006;12:3851-5.

21 Garcia-Carracedo D, Chen ZM, Qiu W, et al. PIK3CA mutations in mucinous cystic neoplasms of the pancreas. Pancreas 2014;43:245-9.

22 Yu J, Sadakari Y, Shindo K, et al. Digital next-generation sequencing identifies lowabundance mutations in pancreatic juice samples collected from the duodenum of patients with pancreatic cancer and intraductal papillary mucinous neoplasms. Gut 2017;66.

23 Garcia-Carracedo D, Turk AT, Fine SA, et al. Loss of PTEN expression is associated with poor prognosis in patients with intraductal papillary mucinous neoplasms of the pancreas. Clin Cancer Res 2013;19:6830-41.

24 Jones $\mathrm{M}$, Zheng Z, Wang J, et al. Impact of next-generation sequencing on the clinical diagnosis of pancreatic cysts. Gastrointest Endosc 2016;83:140-8.

25 Rosenbaum MW, Jones M, Dudley JC, et al. Next-generation sequencing adds value to the preoperative diagnosis of pancreatic cysts. Cancer 2017;125.

26 Tsiatis AC, Norris-Kirby A, Rich RG, et al. Comparison of Sanger Sequencing, Pyrosequencing, and melting curve analysis for the detection of KRAS mutations: diagnostic and clinical implications. J Mol Diagn 2010;12:425-32.

27 Zhong N, Zhang L, Takahashi N, et al. Histologic and imaging features of mural nodules in mucinous pancreatic cysts. Clin Gastroenterol Hepatol 2012;10:192-8.

28 Bosman FT, Carneiro F, Hruban RH. Theise ND. World Health Organization (WHO) classification of tumours of the digestive system. Lyon, France: IARC Press, 2010.

29 Kakar S, Pawlik TM, Allen PJ, et al. Amin MB, ed. AJCC Cancer Staging Manual: Springer Nature, 2017.

30 Nikiforova MN, Wald Al, Roy S, et al. Targeted next-generation sequencing panel (ThyroSeq) for detection of mutations in thyroid cancer. J Clin Endocrinol Metab 2013;98:E1852-E1860.

31 Grasso C, Butler T, Rhodes K, et al. Assessing copy number alterations in targeted amplicon-based next-generation sequencing data. J Mol Diagn 2015;17:53-63.

32 Jimenez RE, Warshaw AL, Z'graggen K, et al. Sequential accumulation of K-ras mutations and p53 overexpression in the progression of pancreatic mucinous cystic neoplasms to malignancy. Ann Surg 1999:230:501.

33 Jiao Y, Shi C, Edil BH, et al. DAXXIATRX, MEN1, and mTOR pathway genes are frequently altered in pancreatic neuroendocrine tumors. Science 2011;331:1199-203.

34 Mukewar S, de Pretis N, Aryal-Khanal A, et al. Fukuoka criteria accurately predict risk for adverse outcomes during follow-up of pancreatic cysts presumed to be intraductal papillary mucinous neoplasms. Gut 2017;66. 